\title{
Assessing background air pollutant concentrations for modelling studies: evaluation of addition equations under Irish conditions
}

\author{
A. Donnelly, B. Broderick \& B. Misstear \\ Department of Civil, Structural and Environmental Engineering, \\ Trinity College Dublin, Ireland
}

\begin{abstract}
Values adopted for background pollutant concentrations can have a significant effect on the accuracy of the final results in air quality modelling studies. In the absence of a reliable method of combining modelled and background concentrations it has been common practice to sum the percentiles or annual means of each contribution to obtain a value for comparison with limit values. This is often not appropriate as in many cases the meteorological conditions producing high concentrations from the source do not correspond to those resulting in high background concentrations. The validity of a number of equations derived in the UK to add background $\mathrm{NO}_{2}$ and $\mathrm{PM}_{10}$ concentrations to modelled stack contributions has been examined for Irish conditions. The equations allow a total percentile concentration to be predicted at a given receptor based on an annual mean background concentration and hourly modelled concentrations. A theoretical point source was modelled using the point source Gaussian plume equation and corresponding meteorological data, and the addition equations applied using monitored background $\mathrm{NO}_{2}$ and $\mathrm{PM}_{10}$ data. The equations were also tested for a line source, modelled using the General Finite Line Source Model (GFLSM). Baseline values were calculated by addition of the relevant hourly or daily background concentration to the modelled concentrations to produce a full year of total hourly or daily concentrations. Percentiles and annual mean values, and corresponding 95\% confidence limits were calculated directly from this data set. Percentile concentrations predicted by each of the equations were compared to the baseline
\end{abstract}


values. It was found that all equations produced values outside the confidence limits, indicative of systematic variation. Certain methods, however, provide an improvement to commonly applied addition methods and may be useful for screening purposes. Results for the line source were poorer than those for the point source. Further research in this area is required to develop more accurate methods for the addition of background concentrations to modelled contributions, particularly in the case of line sources.

Keywords: background concentrations, $\mathrm{NO}_{x}, \quad \mathrm{NO}_{2}, \quad \mathrm{P} \mathrm{M}_{10}, \quad$ modelled concentrations, limit values, percentiles.

\section{Introduction}

Air quality dispersion models are frequently used in the assessment of air quality in urban and rural settings. Commonly used models provide a prediction of concentrations at a given receptor due to the source(s) being modelled. The background concentration must then be added on to the modelled output at the discretion of the air quality modeller. Recent research had shown that the values adopted for background concentrations can have a significant effect on the accuracy of the overall modelling study (Broderick et al. [1], Vardoulakis et al. [2]). In the absence of a reliable method of combining source and background concentrations it has been common practice in Ireland to sum the corresponding percentiles or annual means of each of the background and the source concentrations for comparison to limit values, both to demonstrate compliance with limit values and in the development of environmental impact statements (EIS). This is often not appropriate as in many cases the meteorological conditions producing high concentrations from the line or point source do not correspond to those resulting in high background concentrations.

In an attempt to more accurately determine total concentrations, a number of additional equations were derived in association with the UK Environment Agency to add background concentrations to modelled stack concentrations (Stedman et al. [3, 4], Abbott and Downing [5]). These equations produce a total percentile concentration using modelled annual mean and percentile concentrations and an annual mean background concentration as input. This paper focuses on assessing the validity and applicability of these equations to Irish conditions. A theoretical point source was modelled using the point source Gaussian plume equation and these equations were applied using monitored hourly and daily $\mathrm{NO}_{2}$ and $\mathrm{PM}_{10}$ data from two background sites in Ireland. Hourly and daily meteorological data was obtained from meteorological sites located close to the air monitoring sites. The equations were also tested for the addition of background $\mathrm{NO}_{2}$ concentrations to a modelled line source, modelled using the general finite line source model (GFLSM). Baseline total concentrations were produced through the hourly (or daily) addition of the modelled output to the background concentration. Percentile or annual mean concentrations were then calculated from the combined data set. The results produced by each of the simple methods were compared to these baseline total values. 


\section{Methods}

\subsection{Monitoring sites}

The Environmental Protection Agency (EPA) monitors air quality at 26 stations throughout the country (O'Leary [6]). Pollutants monitored include particulate matter, ozone, $\mathrm{NO}_{\mathrm{x}}, \mathrm{SO}_{2}$, lead, $\mathrm{CO}$ and benzene. For the purpose of this study two background $\mathrm{NO}_{2}$ and $\mathrm{PM}_{10}$ monitoring sites with differing characteristics were chosen. The first site is located in Kilkitt, Co. Monaghan. This is a rural background site and roads in the surrounding area are of low capacity. The site is at an altitude of $240 \mathrm{~m}$ and the closest town is Carrickmacross located $16 \mathrm{~km}$ away. $\mathrm{NO}_{\mathrm{x}}$ is monitored on an hourly basis using chemiluminescence samplers and $\mathrm{PM}_{10}$ is monitored daily using gravimetric samplers. The second site is located at Glashaboy in Co. Cork. The site is situated at an altitude of $70 \mathrm{~m}$ (which is higher than the surrounding area). This is a rural background site (due to the absence of any sources in the direct vicinity and the altitude of the monitor), but due to its proximity to Cork City $(6 \mathrm{~km})$ and a major roadway $(0.5 \mathrm{~km})$ it has urban influences.

Meteorological parameters (hourly wind speed, wind direction and stability class) are monitored by Met Éireann on an hourly basis at a number of countrywide stations. Data from two stations were used to correspond with the air quality monitoring stations. The first station is at Clones in Co. Monaghan and the second at Cork Airport in Co. Cork.

\subsection{Dispersion models}

Two air quality models were developed using Microsoft excel as a user interface, based on simple dispersion laws, a point source Gaussian plume model and a general finite line source model.

\subsubsection{Point source Gaussian plume model}

The Gaussian plume model is a commonly used air pollution model based on the description of the three dimensional concentration field generated by a point source under stationary meteorological and emission conditions (Zannetti [8). For predicting $\mathrm{NO}_{2}$ concentrations including a term for reflection at ground surface (based on the assumption that no pollutant is absorbed by the ground) the equation takes the following form,

$c(x, y, z)=\frac{Q}{2 \pi \sigma_{h} \sigma_{z} \bar{u}} \exp \left[-\frac{1}{2}\left(\frac{y}{\sigma_{h}}\right)^{2}\right] \cdot\left\{\exp \left[-\frac{1}{2}\left(\frac{\left(z_{s}-H\right)^{2}}{\sigma_{z}}\right)\right]+\exp \left[-\frac{1}{2} \frac{(z+H)^{2}}{\sigma_{z}}\right]\right\}$

where $c(x, y, z)$ is the concentration at point $x, y, z$; $\mathrm{Q}$ is the emission rate; $\sigma_{h}$ and $\sigma_{z}$ are the horizontal and vertical standard deviations of the plume concentration spatial distribution (vertical and horizontal dispersion coefficients) calculated based on the downwind distance of the receptor form the source, $d$; $\bar{u}$ is the average wind velocity vector. $\mathrm{H}$ is the assumed height of emission of the plume, including allowance for plume rise. The reflection at ground surface term was 
not included for the modelling of particulate matter as it is assumed to be deposited on the ground when the plume reaches ground level.

Dispersion equations based on the Pasquill stability classes were used to derive $\sigma_{h}$ and $\sigma_{y}$. These equations can be found in Masters [9]. As input the model takes receptor point coordinates, emission rate data, hourly or daily wind speed, wind direction and stability class data. As output the model produces an equivalent set of concentrations at a given receptor.

\subsubsection{General finite line source model}

Luhar and Patil [10] developed a simple General Finite Line Source (GFLSM) model based on the Gaussian diffusion equation which can be used for any orientation of wind direction with the roadway. The model is based on transformation between the wind coordinate system and the line source coordinate system. The final form of the GFLSM is as follows,

$$
\begin{aligned}
& c(x, y, z)=\frac{Q}{2 \sqrt{2 \pi} \sigma_{z} \bar{u}}\left[\exp \left\{-\frac{1}{2}\left(\frac{z-h_{o}}{\sigma_{z}}\right)^{2}\right\}+\exp \right]\left\{-\frac{1}{2}\left(\frac{z+h_{o}}{\sigma_{z}}\right)^{2}\right\}, \\
& \left.\cdot \operatorname{erf}\left|\frac{\sin \theta(l / 2-y)-x \cos \theta}{\sqrt{2} \sigma_{y}}\right|+\operatorname{erf}\left|\frac{\sin \theta(l / 2+y)+x \cos \theta}{\sqrt{2} \sigma_{y}}\right|\right]
\end{aligned}
$$

where $\mathrm{c}$ is the concentration at coordinates $x, y, z$ in the road coordinate system; $\mathrm{Q}$ is the source emission rate per unit length; $\overline{\mathrm{u}}$ is the mean ambient wind speed; $h_{o}$ is the source height ( $=$ road height + plume rise); $\sigma_{z}$ and $\sigma_{y}$ are the dispersion coefficients as before; $\theta$ is the angle between the road and the wind vector (measured anti clockwise with respect to the road). The vertical and horizontal dispersion coefficients are dependent on the downwind distance and were calculated using the Briggs' urban dispersion coefficients (Zannetti [8]).

A simple model was developed using receptor coordinates, road angle (measured clockwise from true north), hourly or daily wind speed, wind direction and stability data, hourly or daily traffic flow, emission factors, link length and link height as input data. As output the model produces an equivalent set of concentrations at a particular receptor.

\subsection{Addition methods}

The following section describes both commonly used addition methods and a selection of the simple methods developed by Abbott and Downing [5] and Stedman et al. [3, 4] which were found to be most accurate.

\subsubsection{Baseline method}

The monitored background concentrations were added hour by hour to the modelled concentrations and the relevant percentile subsequently calculated. This is assumed to be the true value. Hours where monitored data were missing or where the model was unable to calculate the concentration due to low wind speed were left out of the analysis. The oxides of nitrogen emitted from stacks and exhausts are a combination of $\mathrm{NO}_{2}$ and NO. In order to account for the 
limitation of $\mathrm{NO}_{2}$ based on the conversion of $\mathrm{NO}$ to $\mathrm{NO}_{2}$, the following method is used to calculate the $\mathrm{NO}_{2}$ concentration for each hour.

$$
T^{N O 2}=A^{N O 2}+0.05 S^{N O x}+\min \left(0.95 S^{N O x}, A^{O 3}\right)
$$

where $\mathrm{T}$ is the total concentration, $\mathrm{S}$ is the stack contribution and $\mathrm{A}$ is the ambient concentration with the superscript indicating the substance.

In the case of $\mathrm{PM}_{10}$ only daily background data was available. Therefore the model was run using daily meteorological data and each modelled daily value was added directly to the corresponding daily background value.

Due to the non-normality of air pollution data a non-parametric method was used to estimate the percentile concentrations as described by Gilbert [10]. The $\mathrm{n}$ data are ordered to obtain sample order statistics, $x 1<=x 2<=x 3 \ldots<=x n$. To estimate the $p^{\text {th }}$ percentile the value of $\mathrm{x}$ corresponding to $\mathrm{p}$ is required. To estimate this value $k=p(n+1)$ is calculated, and then the estimated $p^{\text {th }}$ percentile, $x_{p}$ is the $k^{\text {th }}$ order statistic. If $k$ is not an integer then linear interpolation is used. In order to determine whether the differences between the baseline method results and the subsequent results obtained from the various methods are due to chance variation or systematic differences, confidence limits were calculated for the baseline percentile values. To estimate the lower and upper $100(1-a) \%$ confidence limits for the $p^{\text {th }}$ percentile the lower limit, 1 and the upper limit, u were computed. These values are order statistics and the corresponding value can easily be found by the same method as for calculating the $p^{\text {th }}$ percentile.

$$
\begin{aligned}
& l=p(n+1)-Z_{1-\alpha / 2}[n p(1-p)]^{1 / 2} \\
& u=p(n+1)+Z_{1-\alpha / 2}[n p(1-p)]^{1 / 2}
\end{aligned}
$$

\subsubsection{Commonly applied methods}

There are a number of methods commonly employed to add the background concentration to the modelled contribution. A conservative method is to add the relevant percentile for the modelled contribution to the relevant percentile for the background contribution (percentile method). In each case the subscript q refers to the percentile of interest.

$$
T_{q}=S_{q}+A_{q}
$$

The following equation is suggested by Stedman et al. [3] to account for the effects of ozone limitation on $\mathrm{NO}_{2}$ concentrations.

$$
T_{q}^{N O 2}=0.05 S_{q}^{N O x}+A_{q}^{N O 2}+\min \left(0.95 S_{q}^{N O x}, A_{q}^{O 3}\right)
$$

Alternatively the annual mean background concentration (annual mean method) or twice the annual mean background concentration (twice annual mean method) is added directly to the percentile modelled contribution. 


$$
T_{q}=S_{q}+A_{m}, 2 A_{m}
$$

For $\mathrm{NO}_{\mathrm{x}}$ the following is suggested by Stedman et al. [3],

$$
T_{q}^{N O_{2}}=A_{m}^{N O 2}, 2 A_{m}^{N O 2}+0.05 S_{q}^{N O x}+\min \left(0.95 S_{q}^{N O x}, A_{q}^{O 3}\right)
$$

\subsubsection{Sum of squares}

The sum of squares method uses the additive properties of the variances of the stack and background concentrations and assumes that the two concentrations are not correlated. It is also assume that the concentration distributions are similar.

$$
T_{q}=S_{m}+A_{m}+\sqrt{\left(S_{q}-S_{m}\right)^{2}+\left(A_{q}-A_{m}\right)^{2}}
$$

The following equation is used for $\mathrm{NO}_{\mathrm{x}}$ emissions

$$
T_{q}^{N O_{2}}=A_{m}^{N O 2}+S_{m}^{N O x}+\sqrt{\left(A_{q}^{N O 2}-A_{m}^{N O 2}\right)^{2}+\left(0.05 S_{q}^{N O x}+\min \left(0.95 S_{q}^{N O x}, A_{q}^{O 3}\right)-S_{m}^{N O x}\right)^{2}}
$$

\subsubsection{Annual mean and twice annual mean stack contributions}

The annual mean and twice annual mean stack contribution methods assume that the stack contribution is small relative to the background concentration and high percentile events are mainly due to elevated background concentrations.

$$
T_{q}=S_{m}, 2 S_{m}+A_{q}
$$

For NOx,

$$
T_{A, q}^{N O_{2}}=A_{q}^{N O 2}+0.05 S_{m}^{N O x}, 0.1 S_{m}^{N O x}+\min \left(0.95 S_{m}^{N O x}, A_{m}^{O 3}\right)
$$

\subsubsection{Maximum annual mean and maximum twice annual mean}

The maximum annual mean and maximum twice annual mean methods use the larger of the annual mean stack method and the annual mean method and the twice annual mean stack method and the twice annual mean method respectively.

\subsubsection{Modified technical guidance method}

The modified technical guidance method is recommended by Stedman et al. [4] to produce a conservative estimate of $\mathrm{NO}_{2}$ for screening purposes. The method assumes that the nitric oxide released immediately reacts with ozone to form $\mathrm{NO}_{2}$. The method is therefore expected to be conservative.

$$
T_{q}^{N O_{2}}=\min \left\{A_{q}^{N O_{2}+O_{3}}+0.05 S_{q}^{N O_{x}}, \max \left[S_{q}^{N O_{x}}+2 A_{m}^{N O_{2}}, A_{q}^{N O_{2}}+2 S_{m}^{N O_{x}}\right]\right\}
$$

The superscript $\mathrm{NO}_{2}+\mathrm{O}_{3}$ refers to the total oxidant concentrations of ozone and $\mathrm{NO}_{2}$. 


\section{Results}

The point source Gaussian model (using a 50m stack) was run for $\mathrm{NO}_{\mathrm{x}}$ at the Kilkitt and Glashaboy sites and for $\mathrm{PM}_{10}$ at the Kilkitt site for two full years of data. The GFLSM model was run for $\mathrm{NO}_{\mathrm{x}}$ at the Kilkitt and Glashaboy sites using two full years of data. In each case the receptor was placed in a location downwind of the stack relative to the prevailing south westerly winds and a location upwind of the stack in order to capture two differing conditions. For the stack emissions, the model was run 6 times using $\mathrm{NO}_{\mathrm{x}}$ emissions ranging from $0.025 \mathrm{~g} / \mathrm{s}$ to $200 \mathrm{~g} / \mathrm{s}$ at each location. Similarly 6 runs were carried out for $\mathrm{PM}_{10}$ with emissions ranging from $0.5 \mathrm{~g} / \mathrm{s}$ to $50 \mathrm{~g} / \mathrm{s}$. For the line source the models were also run 6 times at each location for $\mathrm{NO}_{\mathrm{x}}$. A typical traffic flow was obtained from the N8, a road located in close proximity to the site at Glashaboy. Factors varying between 0.1 and 10 were applied to the traffic data to generate varying data sets.

Using the baseline data and each of the simple methods, the $99.8^{\text {th }}$ percentile of total $\mathrm{NO}_{2}$ concentrations and the $90.41^{\text {st }}$ percentile of total $\mathrm{PM}_{10}$ concentrations were calculated for each emission rate and site. Results were normalised by dividing the relevant percentile value by the corresponding baseline percentile value. A number of measures were used to compare the performance of the various methods. Firstly the percentage of values predicted by each of the methods above the baseline value was noted. Secondly the largest and smallest normalised values given by each of the methods were noted. Finally the number of values falling within the confidence limits was noted.

\subsection{Point source Gaussian plume model}

Figures 1 and 2 show sample results enabling comparison between various methods for predicting the $99.8^{\text {th }}$ percentile $\mathrm{NO}_{2}$ concentration at the Kilkitt and Glashaboy sites. In general the methods tend to produce under predictions of the true concentration when the background is high relative to the source and over predictions when the background is low relative to the source. The percentile method is, as expected conservative and over predicts the true $99.8^{\text {th }}$ percentile concentration on all occasions. However it was found that $50 \%$ of predictions fell outside 95\% confidence limits. The twice annual mean and annual mean methods (commonly employed in Ireland) produce $100 \%$ of values outside the confidence limits and severe underestimations of the true total value, in some cases predicting a total concentration 90\% lower than the actual. The twice annual mean stack and annual mean stack methods are the dominating results for the maximum twice annual mean and the maximum annual mean methods, further highlighting the underestimations occurring using the annual and twice annual mean methods. The sum of squares and ozone methods produced very similar results. In all cases the prediction was conservative and above the baseline value. $85 \%$ of results fell within the confidence limits. The modified technical guidance method produced the highest number of values within the confidence limits (88\%). However under predictions did occur (at $90 \%$ of the true value) and the method is not as conservative as the sum of squares method. 


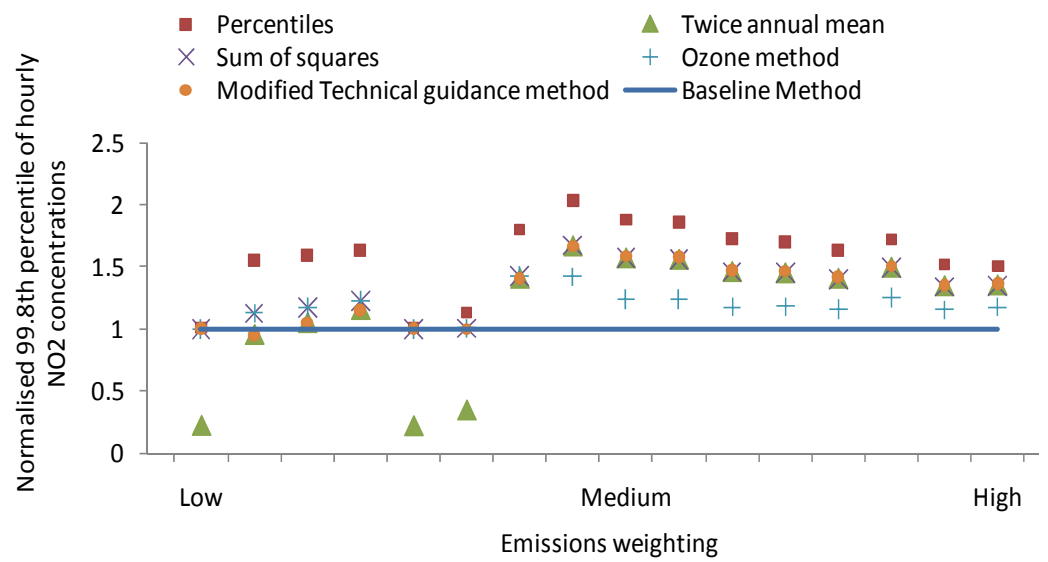

Figure 1: $\quad$ Various addition methods applied to a point source at Kilkitt, $\mathrm{NO}_{2}$.

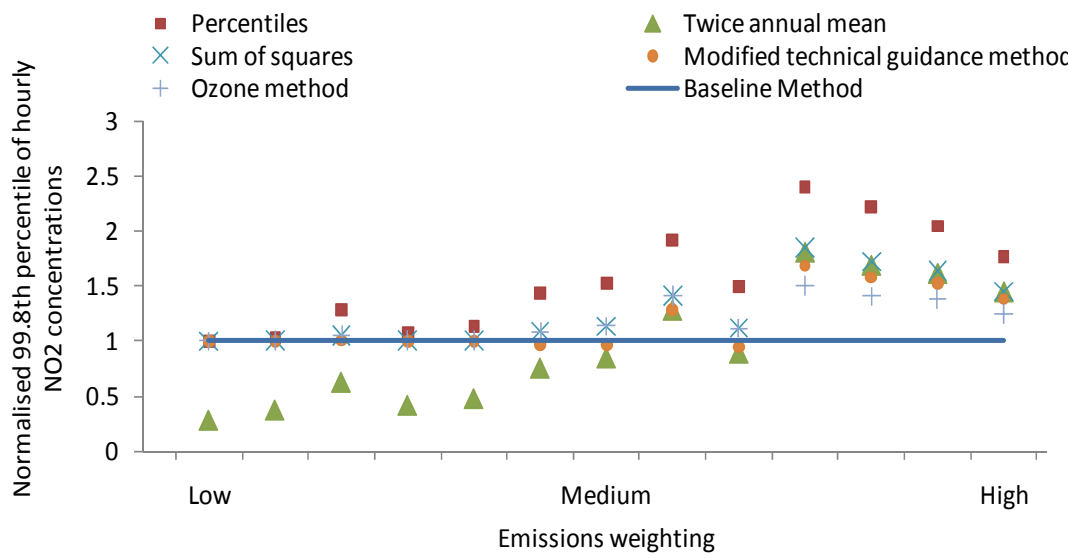

Figure 2: Various addition methods applied to a point source at Glashaboy, $\mathrm{NO}_{2}$.

Figure 3 shows results for $\mathrm{PM}_{10}$ concentrations at Kilkitt. Overall results were poorer and this is most likely due to the higher background $\mathrm{PM}_{10}$ levels relative to the emissions. As can be seen many of the methods tend to produce gross over estimations of the true percentile value for high emission weighting. The twice annual mean stack method produced the greatest number of values within the confidence limits but also resulted in the greatest number of under predictions. The annual mean and the twice annual mean methods, although poor, do perform better for $\mathrm{PM}_{10}$ than for $\mathrm{NO}_{2}$ since background $\mathrm{PM}_{10}$ concentrations have a greater influence on total concentration. The sum of squares method produces the most useful results, conservative on all occasions, but only $50 \%$ of values within the confidence limits. 


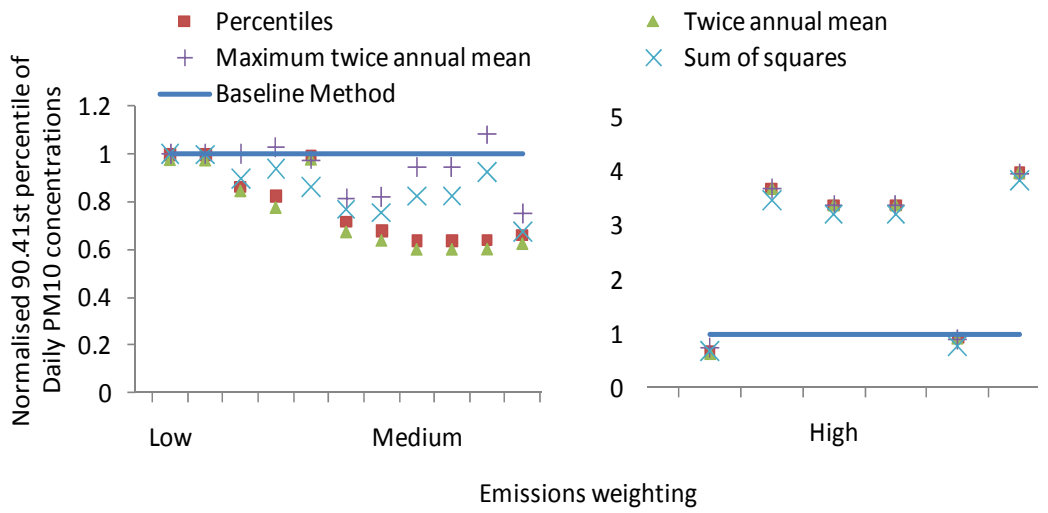

Figure 3: Various addition methods applied to a point source at Kilkitt, $\mathrm{PM}_{10}$.

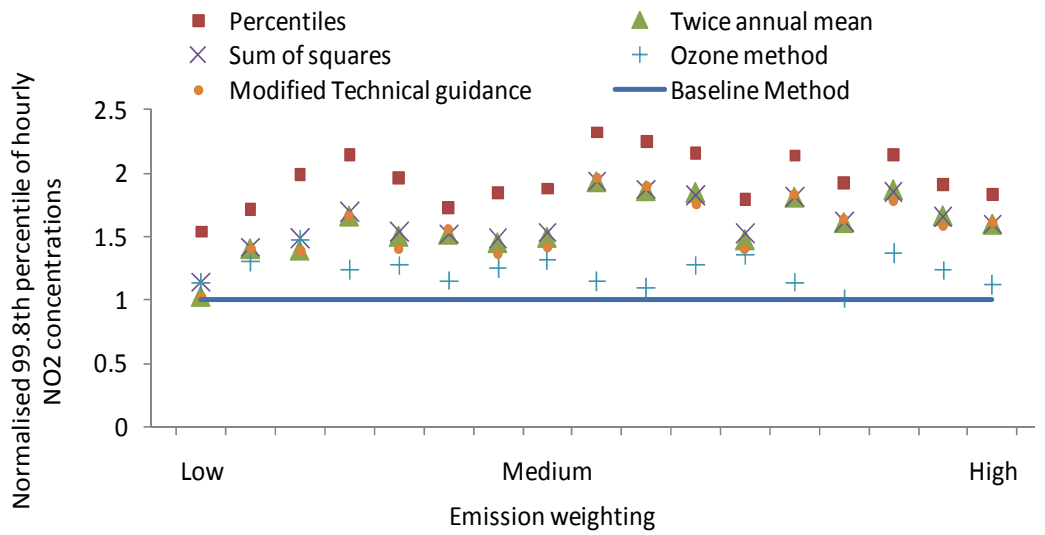

Figure 4: Various addition methods applied to a line source at Kilkitt and Glashaboy, $\mathrm{NO}_{2}$.

\subsection{GFLSM}

Methods applied for predicting the total concentration due to a line source were overall found to produce poorer results as expected. Figure 4 shows the results for the prediction of the $99.79^{\text {th }}$ percentile of hourly $\mathrm{NO}_{2}$ concentrations at Kilkitt and Glashaboy. None of the methods produced an appreciable number of values within the confidence limits. Results at the Glashaboy site (which is influenced to some extent by emissions from a nearby road) were poorer than those at Kilkitt. The twice annual mean stack method produced the highest number of values within the confidence limits. However it produced a large number of predictions below the baseline value. Although many values fall outside the confidence limits, the ozone method produced conservative results without widely varying normalized values. 


\section{Discussion}

Methods commonly employed in Ireland for adding background concentrations to concentrations output for air quality models include the basic percentile, annual mean and twice annual mean methods. It has been found that these methods produce widely varying results and the latter two, in particular can produce severe underestimations of the true concentrations for both point and line source modelling. The percentile method is preferred as it is conservative. However a high number of values fell outside $95 \%$ confidence limits suggesting systematic variation between the values predicted by the method and the true percentile value. The sum of squares or the ozone methods examined here offer improvements for prediction of total $\mathrm{NO}_{2}$ due to a point source and the background concentration. Both methods produce systematically varying results but they tend to be conservative, particularly for high emission levels. They present a significant improvement on the commonly applied annual mean, twice annual mean and percentile methods and are perhaps useful for screening purposes.

Overall it was found that the methods produced poorer results when adding background concentrations to a modelled line source than a stack. It should be stated that the methods were produced specifically for modelled stack concentrations. $\mathrm{NO}_{\mathrm{x}}$ emissions from roads are considerably different in their composition than typical stack releases. The proportion of $\mathrm{NO}_{\mathrm{x}}$ composed of $\mathrm{NO}_{2}$ is generally higher from road emissions than from stack combustion emissions. Furthermore the distribution of concentrations modelled at a particular point is different for line and point source, with differing proportions of low, medium and high pollution events.

The simple methods performed poorly for $\mathrm{PM}_{10}$, producing large under and over estimations, displayed by a large range in normalised values for each simple method. In general it was found that the methods worked better for very low emission weightings. This is because the stack effect on the $90.41^{\text {st }}$ percentile is negligible compared to the background effect and the methods are simply predicting the background $90.41^{\text {st }}$ percentile which is already known. At higher stack emission rates very large over estimations were observed. Overall the sum of squares method produced the most useful results. However a large range in normalised values limits its application. It does produce an improvement on the annual mean, twice annual mean and percentile methods that are commonly employed and should therefore be used in preference to these methods for screening studies.

A further consideration is that no estimation of the annual mean background or percentile concentrations has been made here. Generally in modelling studies the true annual mean or percentile background concentration will not be known. The estimation of such statistics adds a further degree of uncertainty to the analysis. 


\section{Conclusions}

It was found that all methods produced values outside the confidence limits in each case. It can therefore be concluded that there is systematic variation between the results obtained from the baseline method and those obtained from the other methods for both $\mathrm{PM}_{10}$ and $\mathrm{NO}_{2}$. The methods producing the highest number of values within the confidence limits were not necessarily the most useful as many were not sufficiently conservative. The sum of squares and ozone methods for predicting $\mathrm{NO}_{2}$ concentrations due to a point source provide the best results, providing a majority of values within the confidence limits and generally conservative results. Results for the GFLSM were found to be poorer overall but the ozone method provided the best estimate. The sum of squares method produced the best results for predicting total $\mathrm{PM}_{10}$ concentrations. However overall results for $\mathrm{PM}_{10}$ were poorer than those for $\mathrm{NO}_{2}$ and values showed a large range in magnitude. These recommended methods, although producing some erroneous results represent a significant improvement over common practice, whereby the annual mean or twice the annual mean background concentration is added to the relevant percentile of modelled contribution. Such methods although not applicable for detailed air quality studies, may be useful for screening assessments of air quality in an area due to a modelled source.

\section{Acknowledgements}

This work has been funded by the Irish Environmental Protection Agency (EPA) under the STRIVE programme 2007-2013 and the authors would like to thank the EPA for their support. The $\mathrm{NO}_{2}$ and $\mathrm{PM}_{10}$ data was provided by the EPA. The meteorological data at the two sites was obtained from Met Éreann and the traffic flow data was obtained from the National Roads Authority (NRA).

\section{References}

[1] Broderick, B.M., et al., Validation of Air Pollution Dispersion Modelling for the Road Transport Sector under Irish Conditions: Final Report. 2004, Project 2000-LS-6.3-M1). Environmental Protection Agency, Johnstown Castle, Co. Wexford.

[2] Vardoulakis, S., et al., Operational air pollution modelling in the UK-Street canyon applications and challenges. Atmospheric Environment, 2007. 41(22): p. 4622-4637.

[3] Stedman, J., et al., Review of background air quality data and methods to combine these with process contributions: technical modelling aspects. 2006, UK Environment Agency: Bristol.

[4] Stedman, J., et al., Review of background air quality data and methods to combine these with process contributions. 2006, UK Environment Agency: Bristol. 
[5] Abbott, J. and C. Downing, The Addition of Background Concentrations to Modelled Contributions from Discharge Stacks. 2000, UK Environment Agency: Bristol.

[6] O’ Leary, B., Air Quality in Ireland 2007

[7] Key indicators of Ambient Air Quality. 2008, Environmental Protection Agency.

[8] Zannetti, P., Air Pollution Modeling: Theories, Computational Methods, and Available Software. 1990: Van Nostrand Reinhold.

[9] Masters, G.M., Introduction to environmental engineering and science. 1991: Prentice Hall Englewood Cliffs, NJ.

[10] Luhar, A.K. and R.S. Patil, A general finite line source model for vehicular pollution prediction. Atmospheric Environment, 1989. 23(3): p. 555-562.

[11] Gilbert, R.O., Statistical Methods for Environmental Pollution Monitoring. 1987: Wiley. 